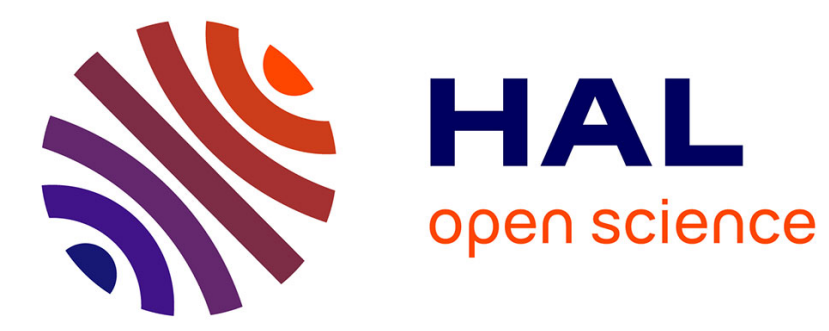

\title{
Optimized magnetic hysteresis management in numerical electromagnetic field simulations
}

\author{
P. Fagan, Benjamin Ducharne, A. Skarlatos
}

\section{To cite this version:}

P. Fagan, Benjamin Ducharne, A. Skarlatos. Optimized magnetic hysteresis management in numerical electromagnetic field simulations. 2021 IEEE International Magnetic Conference (INTERMAG), Apr 2021, LYON, France. pp.1-5, 10.1109/INTERMAG42984.2021.9580043 . hal-03435887

\section{HAL Id: hal-03435887 \\ https://hal.science/hal-03435887}

Submitted on 19 Nov 2021

HAL is a multi-disciplinary open access archive for the deposit and dissemination of scientific research documents, whether they are published or not. The documents may come from teaching and research institutions in France or abroad, or from public or private research centers.
L'archive ouverte pluridisciplinaire HAL, est destinée au dépôt et à la diffusion de documents scientifiques de niveau recherche, publiés ou non, émanant des établissements d'enseignement et de recherche français ou étrangers, des laboratoires publics ou privés. 


\title{
Optimized magnetic hysteresis management in numerical electromagnetic field simulations
}

\author{
P. Fagan ${ }^{1,2}$, B. Ducharne ${ }^{2,3}$, A. Skarlatos ${ }^{1}$ \\ ${ }^{1}$ Université Paris-Saclay, CEA, List, F-91120, Palaiseau, FRANCE. \\ ${ }^{2}$ Laboratoire de Génie Electrique et Ferroélectricité, INSA Lyon, FRANCE. \\ ${ }^{3}$ ELyTMaX UMI 3757, CNRS - Université de Lyon - Tohoku University, International Joint Unit, Tohoku University, Sendai, \\ JAPAN.
}

\begin{abstract}
The treatment of hysteresis in numerical simulations represents major issues as large computational times and significant memory space allocations are required. The memory management of the Jiles-Atherton model is simple, but its integration requires relatively fine temporal discretization to achieve convergence. Oppositely, the Preisach model gives satisfactory results with a coarser temporal grid but requires vast memory space and complex management. The Derivative Static Hysteresis Model (DSHM) is an alternative solution for improved performances. The hysteresis law is considered in a generalized input vector space. An interpolation matrix is constructed with the columns and rows denoting the discrete values of $H$ and $B$ and whose terms stand for the $d B / d H$ slope at the corresponding point. Up to now, the filling step of the DSHM matrix has always been through experimental first-order reversal curves, but getting such experimental data is always complex. In this study, we propose to fill the DSHM matrix alternatively. We use simulated first-order reversal curves obtained from the Jiles-Atherton or the Preisach model, which have been identified using limited experimental data (the first magnetization curve and the major hysteresis cycle).
\end{abstract}

Index Terms - Ferromagnetic hysteresis, space discretization, frequency dependence, simulation optimization.

\section{INTRODUCTION}

In modern society, electromagnetic devices including motors, generators, electromechanical solenoids, relays, loudspeakers can be found in every industrial field. The development of new electromagnetic designs, such as improving and understanding already existing ones, requires precise simulation tools [1][2]. Dedicated commercial software already exist, but they are still very limited, and in this domain, signs of progress are highly expected [3].

Such numerical tools can also be used for the understanding of electromagnetic nondestructive testing (NDT), like the Eddy Current Testing (ECT) [4][5], the Harmonic Analysis [6], the Magnetic Incremental Permeability (MIP) [7][8], the Magnetic Barkhausen Noise (MBN) [9][10], but here again progresses are still to be done for a larger democratization of these simulation tools. Recent developments for the simulation of electromagnetic devices focus on coupling Space Discretization Techniques (SDT), like the Finite Elements Methods (FEM) or the Finite Differences Methods (FDM) to accurate scalar or vector, static or dynamic, hysteresis material laws [11]-[17]. For this material law, the best results come from the extension of a quasi-static hysteresis model to the dynamic behavior resulting from the separation loss technique described by Bertotti in [18].

Running quasi-static hysteresis models brings significant issues as they always require large computational times and memory space allocations. Hysteresis in an SDT resolution means dealing with memory management for each node of the mesh. The allocation of the memory space is linearly proportional to the number of nodes.

Numerous quasi-static hysteresis model descriptions can be found in the literature [19]-[21]. Amongst these, the JilesAtherton (J-A) [22] and the Preisach models [23][24] are by far the most studied hysteresis models. In its original form, the J-A model implementation requires only five parameters, and the memory management is limited to the $t-d t$ value of the magnetization $M$. The $\mathrm{J}$-A model is thus an excellent candidate for integration in an SDT calculation code. Unfortunately, this simple model is subjected to limitations:

convergence is most of the time obtained through fine temporal discretization, which imposes an increased number of samples and simulation time.

Correct simulation of closed minor loops as observed under unsymmetrical excitation field is impossible. The J-A model presents a slow but constant accommodation time, preventing the magnetization trajectory between the turning points of a minor loop from being closed at the end of its course [25].

The Preisach model is much more accurate for the reconstitution of the hysteresis behavior under unsymmetrical excitation (minor loops, first magnetization curve, etc.). Unfortunately, though, correct simulation results imply fine discretization of the Preisach triangle and time-consuming memory management. The signs of many terms in the Preisach triangle are switching at each simulation time step.

In [26], the authors proposed an alternative method for considering the quasi-static hysteresis in SDT simulations. This method, called Derivative Static Hysteresis Model (DSHM), is a data-based reconstruction of the magnetic hysteresis. It shows decent performances under unsymmetrical excitation, works under coarse time discretization, and memory management is found to be very limited. The fact that interpolation in a generalized vector space is used instead of direct branching is also beneficial for the overall algorithm in terms of stability. Up to now, the filling step of the DSHM matrix has always been through a set of experimental first-order reversal curves. It is evident that getting such a lot of experimental data is complex, but in this study, we propose to alternatively fill the DSHM model matrix with a reduced number of experimental data thanks to assumptions also used to parameter the Preisach and the J-A models. The principles of the new method can be detailed as follows:

- The Preisach or the J-A models are identified first using a 
limited number of experimental results (a first magnetization curve and a major loop).

A set of first-order reversal curves is obtained numerically using the Preisach or the J-A model.

The numerical first-order reversal curves are used to fill the DSHM matrix.

The DSHM model is described in the first section of this manuscript, including the alternative method developed for the model matrix construction. In the second part of the manuscript, a benchmark electromagnetic situation chosen to evaluate the model performances is described: the dynamic magnetic field diffusion through the cross-section of a ferromagnetic lamination. This diffusion is solved through FDM resolution, including hysteresis and frequency dependence. The efficiency of the J-A, the Preisach, and the DSHM models are compared in the last section of the manuscript. Conclusions are given regarding the gains and the advantages of the proposed method.

\section{DERIVATIVE STATIC HYSTERESIS MODEL}

\section{A. Experimental observation}

The DSHM model is based on the following assumption: if the magnetic excitation field $H_{i}$ (respectively, the magnetic induction $B_{j}$ ) is the input of the model, the slope $d B / d H_{i, j}$ $\left(d H / d B_{i, j}\right.$, eq. 2) for each point of the $\left(H_{i}, B_{j}\right)$ plane, included in the envelope of the quasi-static major hysteresis loop is supposed to be dependent on only three parameters: the magnetic field $H_{i}$, the induction field $B_{j}$, and the sign of the time derivation of the input excitation $\left(H_{i}\right.$ or $\left.B_{j}\right)$ (see eq. 1).

$$
\begin{aligned}
& {\frac{d B}{d H_{i, j}}}=\frac{d B}{d H_{i, j}}\left(H_{i}, B_{j}, \operatorname{sgn}\left(\frac{d H_{i}}{d t}\right)\right) \\
& {\frac{d H}{d B_{i, j}}}=\frac{d H}{d B_{i, j}}\left(H_{i}, B_{j}, \operatorname{sgn}\left(\frac{d B_{j}}{d t}\right)\right)
\end{aligned}
$$

Fig. 1 below illustrates the DSHM assumption by comparing a first-order reversal curve to a centered cycle in the zone where both could be seen. These experimental results were obtained with a pure iron material, "Telar 57 ARMCO".
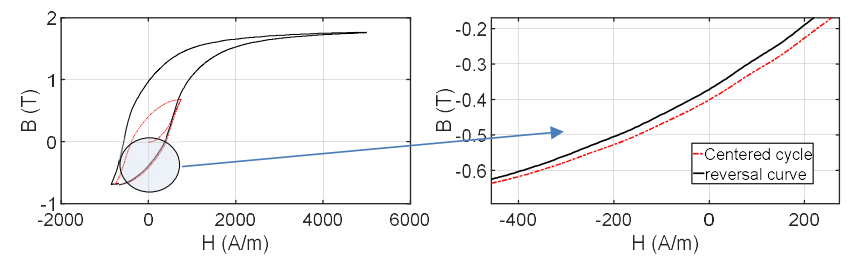

Fig. 1, Illustration of the DSHM assumption for the pure iron Telar 57 ARMCO.

\section{B. Memory storage}

Based on the experimental observations and the assumption commented previously, two matrices can be filled where each column and row represents discretized values of respectively $H$ and $B$. The first matrix is filled with the $d B / d H$ (or $d H / d B$ ) obtained when the sign of the time derivation of $H$ is positive. A set of first-order reversal curves are used to fill this matrix. This matrix behaves like an image of a discretized $(H, B)$ plane. After filling the matrix with all the reversal curves available, many of the matrix terms remain unfilled. If these terms are situated inside the major hysteresis cycle envelope, linear interpolations are done to complete the matrix until one obtains a continuous and smooth distribution of $d B / d H$. Fig. 2 below gives an illustration of the DSHM model matrix and the interpolation process:

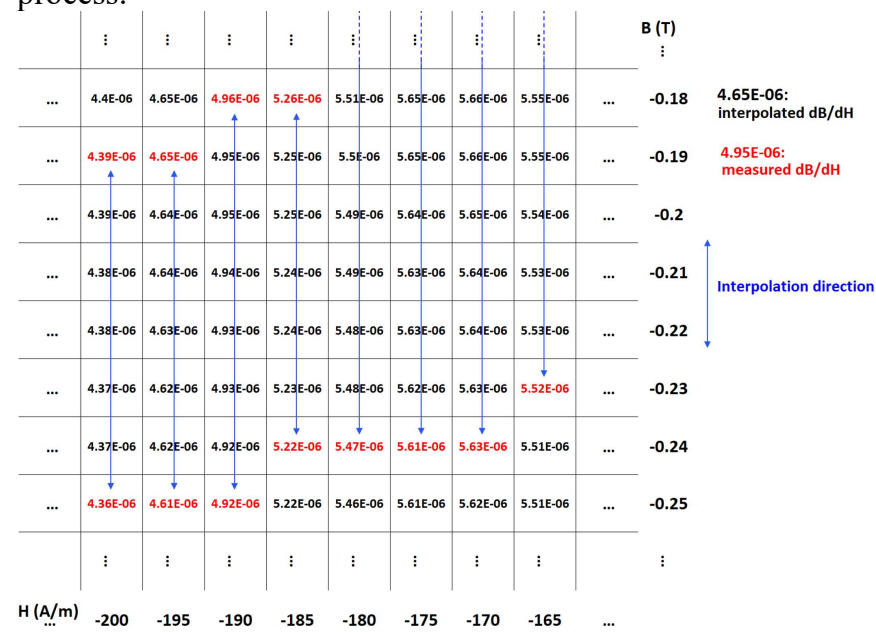

Fig. 2, DSHM interpolation matrix illustration.

The second matrix should be filled with the $d B / d H$ (or $d H / d B$ ) obtained for negative $H$ slopes (decreasing field), but given the point of symmetry of the hysteresis loops, this matrix is redundant. In the simulation, if $H$ decreases, the corresponding $d B / d H$ will be obtained thanks to the " $d B / d H$ increasing $H$ matrix" by multiplying the slope found in the $(-H,-B)$ coordinates of the first matrix with the sign of $(d H / d t)$, (see Eq. 2).

$$
\frac{d B}{d H_{i, j}}\left(H_{i}, B_{j}, \frac{d H_{i}}{d t}<0\right)=-\frac{d B}{d H_{i, j}}\left(-H_{i},-B_{j}, \frac{d H_{i}}{d t}>0\right)
$$

In Fig. 3 below is depicted the 3D plot of the DSHM matrix obtained for the Telar 57 ARMCO.

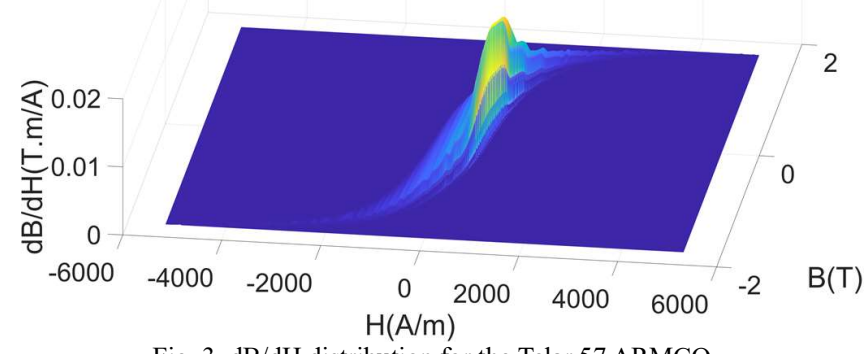

Fig. 3, dB/dH distribution for the Telar 57 ARMCO.

\section{DSHM matrix fill-in}

Numerical results on samples of different compositions show that a minimum of a dozen first-order reversal curves is necessary to fill the $d B / d H$ memory matrix. For exotic materials such as an 80/20 iron-nickel (permalloy) of extreme relative permeability variation, this number can reach 20 . Measuring these reversal curves can be somewhat complicated since it implies applying a magnetic excitation field with a sawtooth shape of varying amplitude (Fig. 4). The substitute option we propose is to numerically simulate the first-order reversal curves using a quasi-static hysteresis model easier to parameterize. Both the JA or the Preisach models can be used successfully for this numerical substitution. In both cases, accurate simulation results can be obtained with experimental data limited to a major 
hysteresis cycle and the first magnetization curve. Fig. 4 below shows some reversal curves simulated with the Preisach model combined with the Biorci \& Pescetti method [27] for the Preisach triangle definition (Telar 57 ARMCO material).

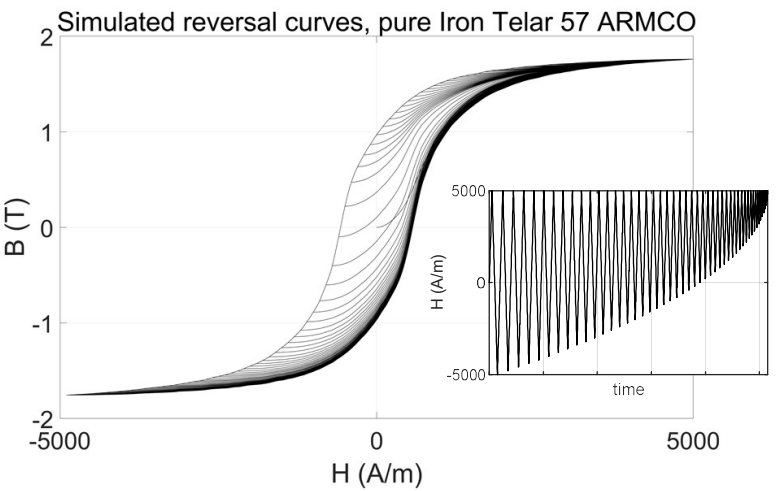

Fig. 4, Simulated reversal curves for the Telar 57 ARMCO.

Once the reversal curves are simulated, the DSHM matrix can be filled. In the case of the Telar 57 ARMCO, a $5 \mathrm{~A} / \mathrm{m}$ step was used for the interpolation matrix column discretization and 0.01 $\mathrm{T}$ for the rows. As the maximum of $H$ was $5200 \mathrm{~A} / \mathrm{m}$ and $B 1.78$ $\mathrm{T}$, the size of the resulting matrix was 357 columns, 2081 rows and a total of 742917 cells

\section{Quasi-static hysteresis simulation}

As Matlab ${ }^{\circledR}$ has been developed for matrix calculation, it is well suited for the implementation of the DSHM model. Fig. 5 below shows a block diagram illustrating the DSHM model principle. For the "H input" version of the DSHM model, $\mathrm{H}(\mathrm{t})$ is already known and set as input. To obtain the related $B(t)$, the corresponding $d B / d H(t)$ has to be determined first. To get this slope, a two-dimensional linear interpolation read on the $d B / d H(H, B)$ interpolation matrix is performed. If $\operatorname{sign}(d H(t) / d t)$ is positive, $H(t)$ and $B(t-d t)$ are used as the scalar coordinate sources for the linear interpolation: $d B / d H(t)(H(t), B(t-d t))$, if not $H(t)$ and $B(t-d t)$ are replaced by $-H(t)$ and $-B(t-d t)$. At this step of the process, $d B / d H(t)$ is consistently positive, but by multiplying it with $\operatorname{sign}(d H(t) / d t)$ it became negative if $H$ decreased and stayed positive otherwise. Once $d B / d H(t)$ is obtained, $B(t)$ is calculated by $H$ integration (see Eq. 3 ).

$$
\begin{gathered}
\text { if } \frac{\boldsymbol{d} \boldsymbol{B}}{\boldsymbol{d} \boldsymbol{H}}>\mathbf{0} \\
B(t)=\int \frac{d B}{d H}\left(H(t), B(t-d t), \frac{d H(t)}{d t}>0\right) d H(t) \\
\text { if } \frac{d B}{d H}<0 \\
B(t)=\int-\frac{d B}{d H}\left(-H(t),-B(t-d t), \frac{d H(t)}{d t}<0\right) d H(t)
\end{gathered}
$$

Finally, $B(t)$ is stored and used as input for the calculation of the next simulation step $B(t+d t)$. Fig. 5 displays both $B(H)$ and $H(B)$ solutions. Switching from a " $B$ input" model to an " $H$ input" model is very simple. To run the " $B$ input" model, the $d B / d H(H, B)$ matrix of the " $H$ input" model is simply replaced by a $d H / d B(H, B)$ matrix, easily obtained by substituting every term of the " $H$ input" matrix by its multiplicative inverse.

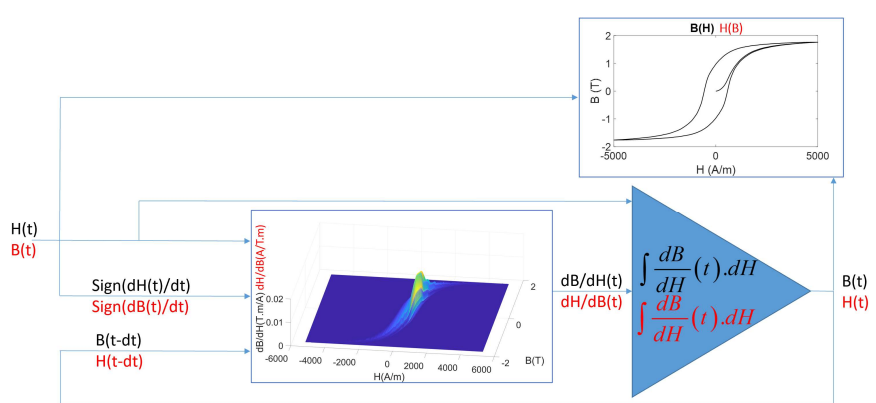

Fig. 5, schematic representation of the DSHM model (black B(H), red H(B)).

\section{E. Single sheet tester characterization and experimental validations}

A dedicated measuring setup has been developed to parameter and validate the numerical method. Epstein frame magnetic sheets (280 mm long, $30 \mathrm{~mm}$ wide) have been characterized. The measuring setup consisted of a single sheet tester with CEI 60404-3 international standards inspiration [28]. The magnetic excitation field $H$ was provided by a 4000 turns coil wound on a high section yoke magnetic circuit. This coil was connected in series with a $10 / 400 \Omega$ variable power resistor whose objective was to avoid a distorted current waveform even under high frequencies excitation. The whole circuit was electrically supplied by a high-voltage KEPCO amplifier BOP 100-4M. Real-time feedback control was ensured from a DEWESoftX2 data acquisition software associated with a SIRIUSif $8 \times \mathrm{CAN}$ data acquisition and generation card. These devices ensured the control of the magnetizing current and the asset of the measured signals. A noise shielded radiometric linear Hall probe (SS94A from Honeywell) positioned ideally as close as possible to the magnetic sample measured the surface magnetic field $H_{\text {surf }}$ over the probe area. A 200-turn pick-up coil surrounded the tested sample was used to measure the magnetic flux. An analogic integration, occurring twice (once for the pick-up coil acquisition and once for the drift correction) was performed to get the flux variation. Before each new measure, we ensured the reproducibility of the result by a complete demagnetization of the tested samples. To validate the DSHM model and the new implementation method on a large scale, a prospective study on magnetic materials of different properties has been carried out. The Telar 57 ARMCO material has been tested first, followed by an iron-nickel permalloy and a FeSi NO electric steel.
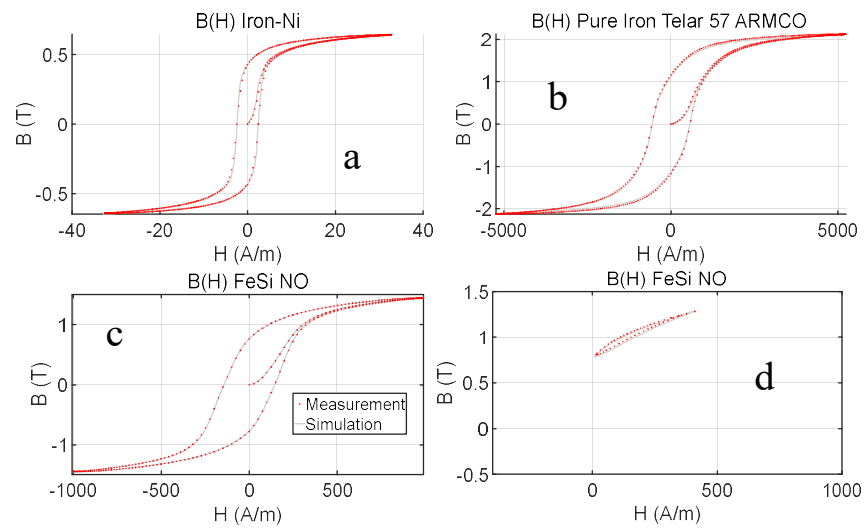


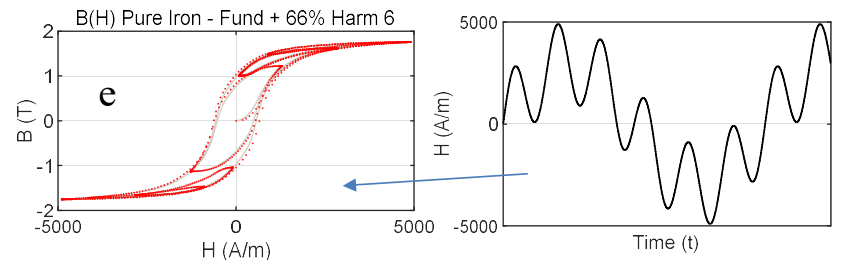

Fig. 6 - a, comparison sim./meas. for the Fe-Ni 80/20, 6 - b, comparison $\mathrm{sim} . /$ meas. for the Pure Iron Telar 57 ARMCO, 6 - c, comparison sim./meas. for the FeSi NO, 6 - $\mathrm{d}$ comparison sim./meas. for FeSi NO under minor loop situation, 6 - e, comparison sim./meas. for Telar 57 ARMCO under a harmonic-type excitation.

The simulation method has been validated over a large window of materials (Fig. 6 - a, b, c). In Fig. 6 - d, e, good simulation results were obtained under unsymmetrical excitation waveform for the FeSi NO (minor loop) and for the Telar 57 ARMCO (harmonic-type excitation).

\section{BENCHMARK TEST: NONLINEAR MAGNETIC FIELD DIFFUSION THROUGH THE CROSS SECTION OF A FERROMAGNETIC LAMINATION}

The ability of the DSHM model and its alternative implementation method to behave correctly under symmetrical and unsymmetrical magnetic excitation waveforms and for different nature of materials have been verified in the previous section. Its performances combined with a numerical solver for the solution of the diffusion problem in ferromagnetic pieces remain to be evaluated. The magnetic field diffusion through the cross-section of a ferromagnetic lamination is strongly nonlinear.

$$
\nabla^{2} \vec{H}=\sigma \cdot \frac{d \vec{B}}{d t}
$$

$\sigma$ is the electrical conductivity. Macroscopic eddy currents due to the dynamic of the external magnetic excitation interfere with microscopic ones linked to the domain wall motions [14][15]. Good simulation results for this diffusive behavior have been obtained by coupling finite differences resolution to frequency-dependent hysteretic material law as detailed in [29][32]. In this experimental situation, $B$ and $H$ are supposed colinear during the magnetization process. This hypothesis allowed to reduce the simulation scheme to scalar quantities. Just like in [30], the equations solved for each node $i$ of the finite difference mesh can be written as follows:

$$
\begin{gathered}
\frac{\partial^{2} H_{i}}{\partial z^{2}}=\sigma \cdot \frac{d B_{i}(t)}{d t} \\
\rho \cdot \frac{d B_{i}(t)}{d t}=H_{i}(t)-f_{\text {quasi-static }}^{-1}\left(B_{i}(t)\right)
\end{gathered}
$$

Here, $f_{\text {quasi-static }}^{1}(B)$ is a quasi-static magnetic field contribution obtained from a quasi-static hysteresis model $H(B)$ and $\rho$ a dynamic constant depending on the tested specimen nature and geometry. The J-A, the Preisach, or the DSHM models described in the first part of this manuscript in their $B(H)$ version were tested for this contribution. The finite differences discretization scheme we opted for is illustrated in Fig. 7 below. The simulation has been carried out in a 1D window using a regular grid for the spatial discretization. The lamination thickness was $0.65 \mathrm{~mm}$ with a discretization step of $0.0016 \mathrm{~mm}$. We assumed the imposed $H_{\text {surf }}$ on top and bottom of the lamination as identical.

The evolution of the local magnetic state as a function of the excitation frequency is depicted in Fig. 8. We illustrate the skin effect, i.e., the increasing eddy current density at the top and bottom layers as the frequency is growing.

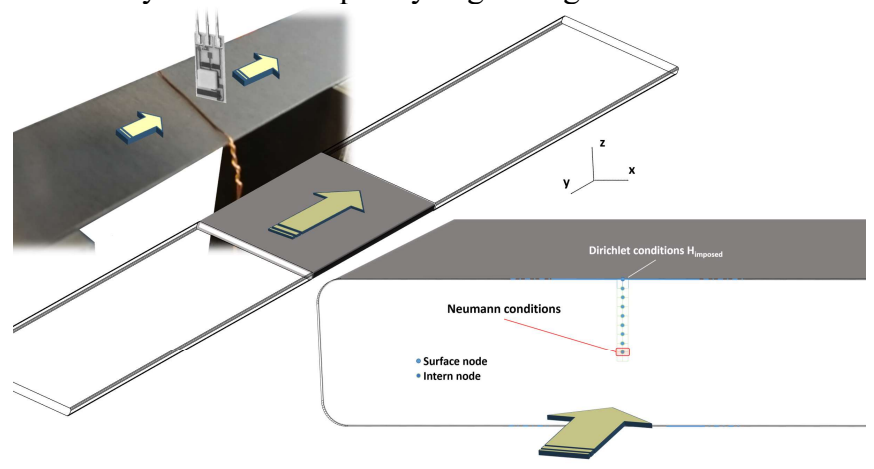

Fig. 7, Finite differences discretization.

The lamination simulated was a FeSi electric steel M400P50, with $\sigma$ being set to $6.410^{6} \mathrm{~S}^{-m^{-1}}$ and $\rho$ to 0.04 .

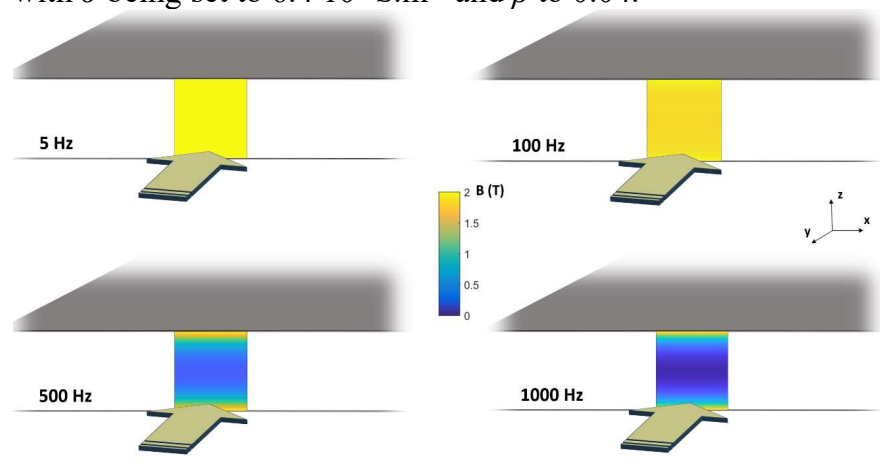

Fig. 8, Magnetic state distribution through the cross section of the lamination.

The result depicted in Fig. 8 below shows the evolution of the hysteresis cycles for different frequencies. $B$ stands for the average of the induction field:

$$
B(t)=\sum_{i=1}^{n} \frac{B_{i}(t)}{n}
$$

$H$ is the imposed tangential component of the magnetic excitation at the specimen surface. The three quasi-static contributions were tested, and the numerical performances were compared in Tab. 1. As observed in Fig. 9, the simulation results obtained using the DSHM model were of reasonable accuracy. In terms of numerical performances, the gain of simulation time was significant $(\div 100)$, as indicated by the computational times provided in Tab. 1. For every test, a suitable timestep was chosen to ensure the stability of the numerical scheme.
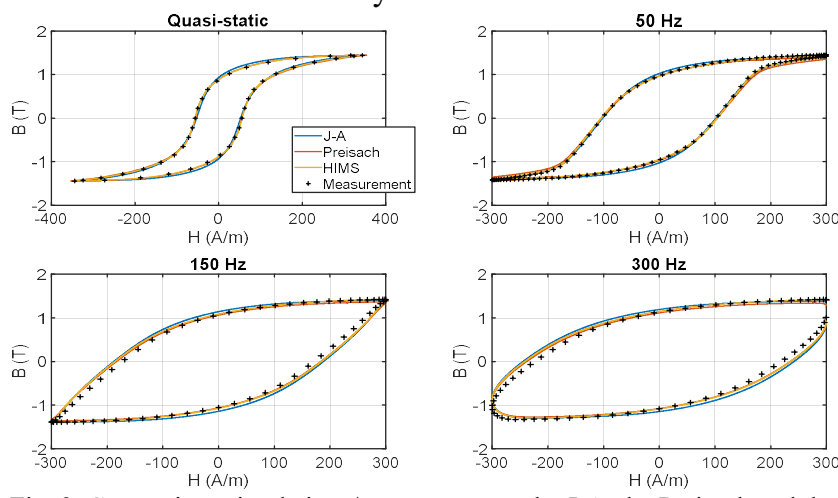

Fig. 9, Comparison simulations/measurements, the J-A, the Preisach and the DSHM are tested successively as quasi-static contribution to check the accuracy of every method. 
Tab. 1 Simulation performances.

\begin{tabular}{|c|c|c|c|}
\hline & J-A & Preisach & DSHM \\
\hline Time (s) & 1096 & 106 & 14 \\
\hline Time discretization & 1000000 & 5000 & 10000 \\
\hline \multicolumn{4}{|l|}{ freq $=150 \mathrm{~Hz}:$} \\
\hline & J-A & Preisach & DSHM \\
\hline Time (s) & 1126 & 44 & 13 \\
\hline Time discretization & 1000000 & 5000 & 10000 \\
\hline \multicolumn{4}{|l|}{ freq $=300 \mathrm{~Hz}:$} \\
\hline & J-A & Preisach & DSHM \\
\hline Time (s) & 1143 & 36 & 14 \\
\hline Time discretization & 1000000 & 5000 & 5000 \\
\hline
\end{tabular}

In the last simulation test, we used the DSHM but with a finer mesh, including 80000 nodes. The same macroscopic behavior (Fig. 9) was observed while the simulation time remained below $21 \mathrm{~s}$, confirming a neat gain in the total simulation time.

\section{CONCLUSION}

The DSHM model is an elegant alternative method for the hysteresis consideration in space discretized simulations. It is characterized by simple memory management and reduced memory allocation. Convergence and precise simulation results can be reached with a relatively coarse temporal discretization. Based on the interpolation of a precalculated matrix hysteresis data, the model is easily reversible and exportable.

To fill the matrix, a set of experimental first-order reversal curves is usually used. It is evident that getting such experimental results can be complicated in many situations, including electromechanical converters design or electromagnetic nondestructive testing, but in this study, we demonstrated that a reduced number of experimental data could fill the DSHM model matrix thanks to assumptions also used to parameter the Preisach and the Jiles-Atherton models. The reversal curves are obtained numerically from the classic simulation methods and used to fill the DSHM matrix.

The DSHM model implemented this way provides correct simulation results even under unsymmetrical waveform situations. Implemented in a nonlinear dynamic finite differences numerical scheme to resolve the magnetic field diffusion through the cross section of a magnetic lamination, it allows to attain considerable speed-up of the simulation process while conserving a very reasonable level of accuracy.

\section{REFERENCES}

[1] P. Zhou, W.N. Fu, D. Lin, S. Stanton and Z.J. Cendes, "Numerical modeling of magnetic devices," IEEE Trans. Mag., vol. 40, iss. 4, pp. $1803-1809,2004$.

[2] O. Bottesi, L. Alberti, R.V. Sabariego, J. Gyselinck, "Finite element smallsignal simulation of electromagnetic devices considering Eddy currents in the laminations," IEEE Trans. Mag., vol. 53, iss. 5, 7400408, 2017.

[3] A.R. Mohd Syaifudin, S.C. Mukhopadhyay, P.L. Yu, "Electromagnetic field computation using COMSOL multiphysics to evaluate the performance of novel interdigital sensors", 2009 App. Electromag. Conf. (AEMC), Kolkata India, 2009.

[4] T. Takagi, H. Huang, H. Fukutomi, J. Tani, "Numerical evaluation of correlation between crack size and eddy current testing by a very fast simulation," IEEE Trans. Mag., vol. 34, iss. 5, pp. 2581 - 2584, 1998.

[5] B. Gupta, B. Ducharne, G. Sebald, T. Uchimoto, "A space discretized ferromagnetic model for nondestructive eddy current evaluation," IEEE Trans. Mag., vol. 54, iss. 3, pp. 1-4, 6200204, 2018.

[6] G. Dobmann, H. Pitsch, "Magnetic tangential field-strength-inspection, a further NDT tool for 3MA", Nondestructive characterization of materials, pp. 636-643, 1989.

[7] H.E. Chen, S. Xie, H. Zhou, Z. Chen, T. Uchimoto, T. Takagi, Y Kensuke, "Numerical simulation of magnetic incremental permeability for ferromagnetic material", Proc. $16^{\text {th }}$ Int. Symp. on App. Elec. And Mech. (ISEM 2013), vol. 45, n¹-4, pp. 379-386, 2014

[8] B. Gupta, B. Ducharne, T. Uchimoto, G. Sebald, T. Miyazaki, T. Takagi, "Physical Interpretation of the Microstructure for aged $12 \mathrm{Cr}-\mathrm{Mo}-\mathrm{V}-\mathrm{W}$ Steel Creep Test Samples based on Simulation of Magnetic Incremental Permeability," J. of Mag. And Mag. Mat., vol. 486, 165250, 2019.

[9] B. Gupta, B. Ducharne, T. Uchimoto, G. Sebald, T. Miyazaki, T. Takagi, "Nondestructive testing on creep degraded $12 \% \mathrm{Cr}-\mathrm{Mo}-\mathrm{W}-\mathrm{V}$ Ferritic test samples using Barkhausen noise," J. of Mag. And Mag. Mat., vol. 498, 166102,2019

[10] B. Ducharne, MQ. Le, G. Sebald, PJ. Cottinet, D. Guyomar, Y. Hebrard, "Characterization and modeling of magnetic domain wall dynamics using reconstituted hysteresis loops from Barkhausen noise," J. of Mag. And Mag. Mat., vol. 432, pp. $231-238,2017$.

[11] M. Kuczmann, A. Iványi, "The Finite Element Method in Magnetics", Budapest, Academic Press, 2008.

[12] J. Saitz, "Newton-Raphson method and fixed-point technique in finite element computation of magnetic fields problems in media with hysteresis," IEEE Trans. Magn., vol. 35, pp. 1398-1401, 1999.

[13] F. Henrotte, "Modeling ferromagnetic materials in 2D finite element problems using Preisach's model," IEEE Trans. Magn., vol. 28, pp. 2614 2616, Sept. 1992.

[14] B. Ducharne, P. Tsafack, Y.A. Tene Deffo, B. Zhang, G. Sebald, "Anomalous fractional magnetic field diffusion through the cross-section of a massive toroidal ferromagnetic core," Com. in Nonlin. Sci. and Num. Sim., vol. 92, 105450, 2020

[15] B. Ducharne, Y.A. Tene Deffo, B. Zhang, G. Sebald, "Anomalous fractional diffusion equation for magnetic losses in a ferromagnetic lamination," The Eur. Phys. J. Plus, 135:325, 2020.

[16] M. Petrun and S. Steentjes, "Iron-Loss and Magnetization Dynamics in Non-Oriented Electrical Steel: 1-D Excitations Up to High Frequencies," IEEE Access, vol. 8, pp. 4568-4593, 2020.

[17] P. Rasilo, E. Dlala, K. Fonteyn, J. Pippuri, A. Belahcen, A. Arkkio, "Model of laminated ferromagnetic cores for loss prediction in electrical machines," IET Digital library, vol. 5, iss. 7, pp. 580 - 588, 2011.

[18] G.Bertotti, "hysteresis in Magnetism," Academic Press, San Diego, USA, 1998.

[19] H. Hauser, "Energetic model of ferromagnetic hysteresis: isotropic m agnetization," J. of App. Phys., vol. 96, n5, 2004.

[20] J. Tellinen, "A simple scalar model for magnetic hysteresis," IEEE Trans. Mag., vol. 34, no. 4, pp. 2200-2206, 1998.

[21] M.L. Hodgdon, "Applications of a theory of ferromagnetic hysteresis," IEEE Trans. Magn., vol. 24, pp. 218-221, 1988.

[22] D.C. Jiles, D.L. Atherton, "Theory of ferromagnetic hysteresis," J. App. Phys., 55, pp. 2115, 1984

[23] F. Preisach, "Über die magnetische Nachwirkung," Zeitschrift für Physik, 94, pp. 277-302, 1935.

[24] I.D. Mayergoyz "Mathematical models of hysteresis," Phys. Rev. Lett, 56, pp. $1518-21,1986$

[25] A. Benabou, JV. Leite, S. Clénet, C. Simão, N. Sadowski, "Minor loops modelling with a modified Jiles-Atherton model and comparison with the Preisach model," J. of Mag. Mag. Mat., vol. 320, n²0, pp. 1034-1038, 2008.

[26] R. Scoretti, R. Sabariego, F. Sixdenier, B. Ducharne, M.A. Raulet, "Integration of a new hysteresis model in the finite elements method", Proc. of COMPUMAG-SYDNEY, 8 Mat. Mod., 11 Num. Tec., 2011.

[27] G. Biorci, D. Pescetti, "Analytical Theory of the behaviour of ferromagnetic materials," Il N. Cim., vol .7, n6, pp 829-843, 1958.

[28] IEC 60404-3, "Magnetic materials - Part 3: Methods of measurement of the magnetic properties of electrical steel strip and sheet by means of a single sheet tester," Int. Elect. Com., April 2010.

[29] O. Maloberti, G. Meunier, A. Kedous-Lebouc, "Hysteresis of Soft Materials Inside Formulations: Delayed Diffusion Equations, Fields Coupling, and Nonlinear Properties," IEEE Trans. Mag., Vol. 44, iss. 6, pp. $914-917,2008$

[30] M. A. Raulet, B. Ducharne, J.P. Masson, and G. Bayada, "The magnetic field diffusion equation including dynamic hysteresis: a linear formulation of the problem," IEEE Trans. Mag., vol. 40, n² 2, pp. $872-$ 875,2004

[31] B. Ducharne, G. Sebald, D. Guyomar, G. Litak "Dynamics of magnetic field penetration into soft ferromagnets," J. of App. Phys., pp. 243907, 2015.

[32] S. Zirka, Y.I. Moroz, F. Marketos, A.J. Moses, "Viscosity-based magnetodynamic model of soft magnetic materials," IEEE Trans. Mag., 42(9), pp. 2121-2132, 2006. 